\title{
Theoretical modeling and design of photonic structures in zeolite nanocomposites for gas sensing. Part II: Volume gratings
}

Dervil Cody

Technological University Dublin, dervil.cody@tudublin.ie

Izabela Naydenova

Technological University Dublin, izabela.naydenova@tudublin.ie

Follow this and additional works at: https://arrow.tudublin.ie/cieoart

Part of the Atomic, Molecular and Optical Physics Commons, and the Optics Commons

\section{Recommended Citation}

D. Cody and I. Naydenova, "Theoretical modeling and design of photonic structures in zeolite nanocomposites for gas sensing. Part II: volume gratings," J. Opt. Soc. Am. A 35, 12-19 (2018)

This Article is brought to you for free and open access by the Centre for Industrial and Engineering Optics at ARROW@TU Dublin. It has been accepted for inclusion in Articles by an authorized administrator of ARROW@TU

Dublin. For more information, please contact

arrow.admin@tudublin.ie, aisling.coyne@tudublin.ie, gerard.connolly@tudublin.ie.

Funder: Technological University Dublin 


\title{
Theoretical modelling and design of photonic structures in zeolite nanocomposites for gas sensing: part II - volume gratings
}

\author{
D. CODY, ${ }^{1}$ I. NAYDENOVA ${ }^{1 *}$ \\ ${ }^{1}$ Centre for Industrial and Engineering Optics, School of Physics and Clinical and Optometric Sciences, College of Sciences and Health, Dublin \\ Institute of Technology, Kevin Street, Dublin 8, Ireland. \\ *Corresponding author: izabela.naydenova@dit.ie
}

Received XX Month XXXX; revised XX Month, XXXX; accepted XX Month XXXX; posted XX Month XXXX (Doc. ID XXXXX); published XX Month XXXX

\begin{abstract}
The suitability of holographic structures fabricated in zeolite nanoparticle-polymer composite materials for gas sensing applications has been investigated. Theoretical modelling of the sensor response (i.e. change in hologram readout due to a change in refractive index modulation or thickness as a result of gas adsorption) of different sensor designs was carried out using Raman-Nath theory and Kogelnik's Coupled Wave Theory. The influence of a range of parameters on the sensitivity of holographically-recorded surface and volume photonic structures has been studied, namely hologram geometry, hologram thickness and spatial frequency, reconstruction wavelength, and zeolite nanoparticle refractive index. From this, the optimum fabrication conditions for both surface and volume holographic gas sensor designs have been identified. Here in part 2, results from modelling of the influence of design on the sensor response of holographically-recorded volume grating structures for gas sensing applications are reported.
\end{abstract}

OCIS codes: $\underline{050.0050}$ Diffraction and gratings; $\underline{280.0280}$ Remote sensing and sensors; $\underline{220.0220}$ Optical design and fabrication

http://dx.doi.org/

\section{INTRODUCTION}

In Part 1 [1], the state of the art and ongoing research in holographic gas sensors was reviewed. Results from theoretical modelling were presented on the optimisation of the sensor response of surface relief grating (SRG) type zeolite-composite holographic gas sensors in both thin and thick regimes using the Raman-Nath and Bragg diffraction theories respectively. The effect of the SRG refractive index modulation (i.e. zeolite refractive index) surface relief amplitude, spatial frequency and reconstruction wavelength on the sensor response of the zeolite composite SRG-based gas sensor was reported.

In this paper, this process to determine the theoretically ideal fabrication conditions for holographic gas sensors has been extended to volume gratings (VGs). The sensitivity of the holographic sensor will depend largely on the extent to which the refractive index of the material changes, and/or on the ability of the material to undergo dimensional changes by shrinking/expanding. Bragg diffraction theory has been applied to investigate the influence of a range of parameters (refractive index modulation, hologram spatial frequency, thickness, reconstruction wavelength) on the response of transmission and reflection mode zeolite-composite VGs to changes in both grating thickness $(\Delta d)$ and refractive index modulation $(\Delta(\Delta n))$ due to gas absorption. The optimum design for a VG-based gas sensor has been identified, and the advantages and disadvantages to this approach are discussed.

\section{THEORY}

\section{A. Principle of operation of VG-based sensors}

As discussed in Part 1, the literature shows that transmission and reflection mode VGs can successfully be used for detection of both liquid and gaseous analytes [2-15]. VGs consist of periodic variations in refractive index throughout the thickness of the layer. Unlike for SRGs where the grating undergoes negligible changes in thickness, $d$, due to gas absorption, VGs can experience swelling even at small analyte concentrations as the analyte molecules permeate the volume of the layer. Therefore, the influence of changes in both $\Delta n$ and $d$ of zeolitecomposite VG sensors are considered here. Porous zeolite nanoparticles are periodically redistributed within a porous host polymer matrix during holographic recording in accordance with the imposed interference pattern of light. The periodicadsorption of gas molecules in regions of high zeolite concentration will result in a change in both $\Delta n$ and $d$ of the grating; for transmission VGs this is measurable as a change in the diffraction efficiency, $\eta$, whereas for reflection $V G$ s a visual change in the hologram colour due to a shift in the reconstruction wavelength, $\lambda_{r}$, will be obtained. The possibility to produce sensor readout in the form of visible colour changes is a major advantage of VG-based sensors. A possible disadvantage of VG-based gas sensors is that the gas 
molecules are required to permeate the polymer matrix layer, which may impede sensor response time and reversibility, if it is desired.

Sensors based on VGs are not limited in the same way as SRGs; gratings with thickness up to hundreds of microns can be readily fabricated. This widens the range of spatial frequencies and thicknesses that can be explored for optimisation of volume structure devices and ensures that sensors operate in the thick Bragg regime, allowing for $\eta$ values of up to $100 \%$ and, therefore, potentially increased sensitivity in comparison to thin regime devices for which $\eta$ is limited to $33 \%$. E.g. for $\lambda_{r}$ of $532 \mathrm{~nm}$, the minimum allowable spatial frequency for a $50 \mu \mathrm{m}$ thick grating to be classified as thick (i.e. $Q \geq 10$ ) is 300 lines $/ \mathrm{mm}$.

\section{Transmission mode VGs for gas sensing}

The gas sensing mechanism for transmission mode VGs is demonstrated in fig. 1. As gas molecules permeate the matrix, the VG medium will swell resulting in changes in $d$. It is assumed, however, that swelling effects occurring in the plane of the grating are negligible, as has been confirmed experimentally, so $\Lambda$ remains practically unchanged [16]. In zeolite-composite media, the zeolite nanoparticles are spatially redistributed within the transmission grating, producing a refractive index modulation $\Delta n=n_{p}-n_{z}$, where $n_{p}$ is the refractive index of the polymer matrix. The value of $n_{z}$ will change due to adsorption of gas molecules to the zeolite surface and pores, resulting in a variation in the grating $\Delta n$. Changes in both $\Delta n$ and $d$ are detectable as a change in $\eta$.

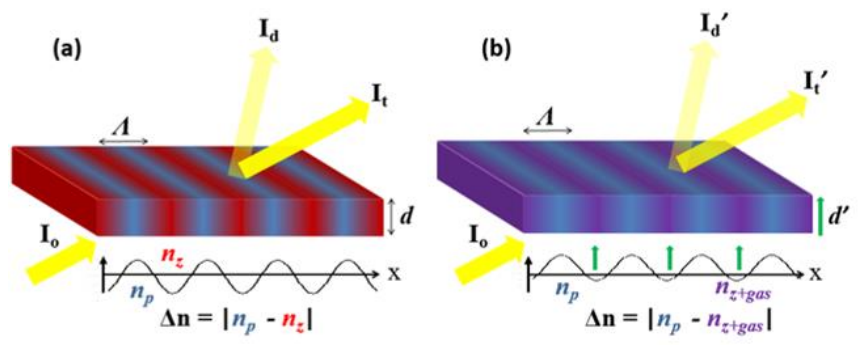

Fig. 1. Zeolite-doped transmission mode VG with thickness $d$ and fringe spacing $\Lambda$ (a) before and (b) after gas exposure.

\section{Reflection mode VGs for gas sensing}

The sensing mechanism for reflection mode VGs is demonstrated in fig. 2. As for transmission mode VGs, the value of $n_{z}$ is altered after gas exposure, resulting in a change in the overall value of the grating $\Delta n$. Swelling also plays a significant role for reflection holograms where the hologram planes are formed parallel to the surface of the sensor material and at higher frequencies. Volume gratings in the reflection regime can be recorded for spatial frequencies up to approximately 5640 lines $/ \mathrm{mm}$ at a wavelength of $532 \mathrm{~nm}$, assuming $n_{\mathrm{p}} \sim 1.5$. Hence, even small changes in $d$ will have a significant impact on the VG period. Changes in $\Delta n$ and $d$ are clearly detected as a visual change in $\lambda_{r}$.
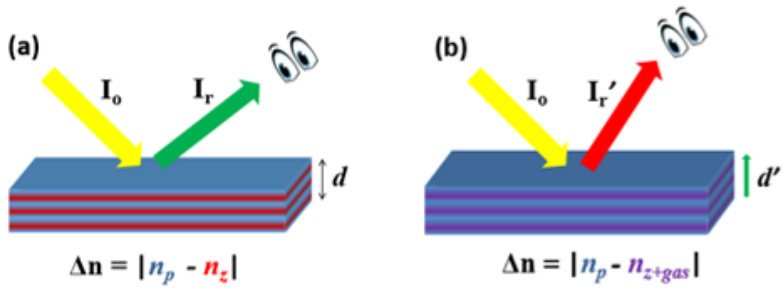

Fig. 2. Zeolite-doped reflection mode VG with thickness $d$ (a) before and (b) after gas exposure.

\section{B. Process of VG sensor design}

The different stages of VG sensor development are shown in fig. 3. Step 1 is obviously to identify the target analyte which is of interest. Step 2 is to choose a functionalising material (in this case, type of zeolite nanoparticle) which is selective and sensitive to the target analyte, as well as compatible with the support or host structure (i.e. polymer grating). As in Part 1 for SRG-based sensors, optimum sensor function is achieved when the smallest possible change in the initial $\Delta n$ or $d$ of the functionalised VG due to gas adsorption will produce a measurable change in the sensor output i.e. diffraction efficiency or reconstruction wavelength, depending on the geometry. In addition, the functionalising material used must meet other requirements for VG sensors which do not apply to SRG-based sensors. The periodic redistribution of the functionalising material throughout the host matrix via holography or some other method must be possible. This places restrictions of the size of the functionalising particles, which must be small enough to diffuse within the VG during holographic recording.

Step 3 involves identification of the optimum VG sensor platform. As seen in section $2 \mathrm{~A}$, due to the fact that the gas molecules must permeate the volume of the layer to adsorb to the redistributed zeolites, polymerbased VG sensors are subject to changes in both $d$ and $\Delta n$ due to gas adsorption. This is problematic as, unlike for SRG-based sensors, two factors are influencing the readout of the VG sensor device. This should be considered when selecting the functionalising and host materials; ideally they should be chosen so as to reduce effects from either swelling or changes in $\Delta n$, in the case that these effects may counteract each other. Selection of the sensor platform also involves determining whether to fabricate a transmission of reflection mode VG sensor. This will depend on the application-specific readout requirements i.e. change in hologram diffraction efficiency or reconstruction wavelength.

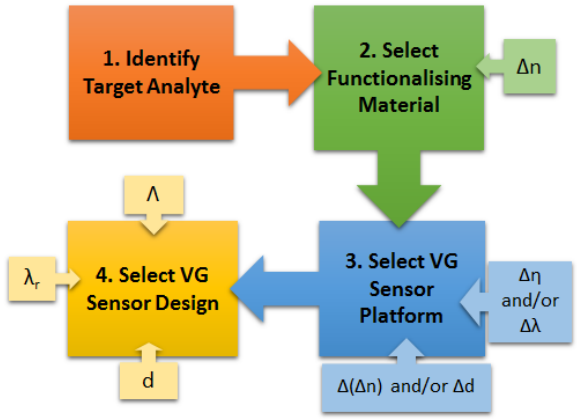

Fig. 3. Flowchart of steps involved in VG sensor design.

\section{Description of theoretical model and equations}

In the literature there are two different parameters that are used to determine whether a grating operates as a thick or a thin grating. They are both derived after evaluation of the solutions of the wave equation and the amount of light transferred to the higher orders of diffraction. As in part 1 [1], both the Klein-Cook $Q$ parameter [17] and the Moharam and Young $\rho$ parameter [18] were applied in order to ensure the VGs are operating in the thick regime:

$$
\begin{gathered}
Q=\frac{2 \pi \lambda_{r} d}{n \Lambda^{2}} \\
\rho=\frac{\lambda_{r}^{2}}{\Lambda^{2} n \Delta n}
\end{gathered}
$$

where $n$ is the recording medium refractive index, $\Delta n$ is the refractive index modulation and $\Lambda$ is the grating period. In order for the VG gratings to be considered strictly thick, $Q \geq 10$ and $\rho \geq 10$ are required. While generally speaking the two criteria classify the gratings in the same category, deviations do occur, for example for very large refractive index modulations where gratings operating typically as thick gratings (characterised by large $Q$ factor) could produce strong multiple orders of diffraction (predicted as thin by the small $\rho$ factor). 
Thick phase gratings exhibit Bragg behaviour and produce only one diffracted beam. Maximum $\eta$ is obtained when the reconstruction beam is incident on the grating at a particular angle of incidence, $\theta_{B}$, called the Bragg angle, given by the Bragg equation [19]:

$$
\lambda_{r}=2 \Lambda \sin \theta_{B}
$$

\section{Transmission mode VGs for gas sensing}

For thick holographic transmission gratings, $\eta$ is defined by Kogelnik's couple wave theory [20] as:

$$
\eta=\sin ^{2}\left(\frac{\varphi}{2}\right)
$$

where $\varphi$ is the grating phase, defined as:

$$
\varphi=\frac{2 \pi \Delta n d}{\lambda_{r} \cos \theta_{B}}
$$

For transmission VGs, the change in $\eta$ relative to the change in $\varphi$ due to gas adsorption has been modelled by taking the partial derivative of eqn. 3 with respect to $\varphi$ :

$$
\frac{\partial(\eta)}{\partial(\varphi)}=\frac{1}{2} \sin (\varphi)
$$

The individual effects of the change in $\eta$ relative to the change in $\Delta n$ and $d$ is found by taking the partial derivative of eqn. 3 with respect to $\Delta n$ and $d$ respectively:

$$
\begin{aligned}
& \frac{\partial(\eta)}{\partial(\Delta n)}=\frac{\pi d}{\lambda_{r} \cos \theta_{B}}\left[\sin \left(2 \frac{\pi \Delta n d}{\lambda_{r} \cos \theta_{B}}\right)\right] \\
& \frac{\partial(\eta)}{\partial(d)}=\frac{\pi \Delta n}{\lambda_{r} \cos \theta_{B}}\left[\sin \left(2 \frac{\pi \Delta n d}{\lambda_{r} \cos \theta_{B}}\right)\right]
\end{aligned}
$$

\section{Reflection mode VGs for gas sensing}

From Kogelnik's coupled wave theory for thick holographic reflection gratings, $\eta$ is defined as:

$$
\eta=\tanh ^{2}\left(\frac{\varphi}{2}\right)=\tanh ^{2}\left(\frac{\pi \Delta n d}{\lambda_{r} \cos \theta_{B}}\right)
$$

The change in $\eta$ relative to the change in $\varphi$ due to gas adsorption has been modelled by taking the partial derivative of eqn. 8 with respect to $\varphi$ :

$$
\frac{\partial(\eta)}{\partial(\varphi)}=\tanh \left(\frac{\varphi}{2}\right)\left[1-\tanh ^{2}\left(\frac{\varphi}{2}\right)\right]
$$

The change in $\lambda_{r}$ resulting from the changes in $\Delta n$ and $d$ due to gas exposure is found by taking the partial derivatives of $\lambda_{r}$ in eqn. 9:

$$
\begin{aligned}
& \frac{\partial\left(\lambda_{r}\right)}{\partial(\Delta n)}=\frac{\pi d}{\tanh ^{-1}(\sqrt{\eta}) \cos \theta_{B}} \\
& \frac{\partial\left(\lambda_{r}\right)}{\partial(d)}=\frac{\pi \Delta n}{\tanh ^{-1}(\sqrt{\eta}) \cos \theta_{B}}
\end{aligned}
$$

Using eqns. 5-7 for transmission VGs and eqns. 9-11 for reflection VGs, the sensitivity of the VG-based sensors (i.e. the change in readout $\eta$ or $\lambda_{r}$ with variation of $\varphi, \Delta n$ and $d$ due to analyte adsorption) can be determined as a function of a range of the grating characteristics, namely initial grating refractive index modulation $(\Delta n)$, spatial frequency, grating thickness $(d)$ and reconstruction wavelength $\left(\lambda_{r}\right)$. From this, the optimum design for zeolite-composite sensors based on transmission and reflection mode VGs has been determined. Additional information is also obtained as to the relative sensitivity of gas sensors based on changes in $\Delta n$ or $d$.

\section{RESULTS AND DISCUSSION}

The different VG sensor configurations which have been subjected to theoretical analysis in order to determine the optimum sensor design in each case are outlined in fig. 4 .

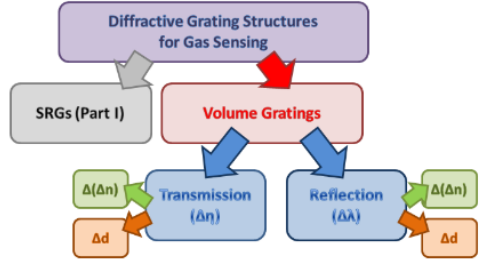

Fig. 4. Schematic of the different sensor configurations considered for theoretical modelling.

\section{A. Modelling results for transmission mode VGs}

The sensor response (i.e. change in $\eta$ as a function of change in $\Delta n$ and $d$ of the grating due to gas absorption) of transmission mode VGs has been modelled using Kogelnik's Coupled Wave theory as described in section 2C.1. The influence of the initial $\Delta n$, spatial frequency, thickness, d, and reconstruction wavelength, $\lambda_{r}$, on the VG sensor response has been investigated as a function of changes in both $\Delta n$ and $d$.

\section{Initial phase difference, $\varphi$}

Let us first consider the general case for transmission mode VG-based gas sensors where the sensor output is varied due to changes in the phase difference $\varphi$ as a result of gas adsorption. The diffraction efficiency of a transmission grating as a function of initial phase difference $\varphi$, given by eqn. 3 , is shown in fig. 5(a). The relationship is sinusoidal, with peaks and troughs corresponding to maximum $(100 \%)$ and minimum (0\%) diffraction efficiency observed at $\varphi=0,3.15,6.3$, 9.45, etc. radians

Fig. 5(a) also shows the change in sensor diffraction efficiency relative to the change in $\varphi$ due to gas adsorption (given by eqn. 5) for different values of initial phase different introduced by the grating. When the initial phase difference $\varphi$ value is equal to $3.15,6.3,9.45$, etc. radians, the value of $\frac{\partial(\eta)}{\partial(\varphi)}$ goes to zero, and so the change in $\varphi$ required to produce a measurable change in diffraction efficiency tends to infinity. This is clearly seen in fig. 5(b), which shows the change in $\varphi$ due to gas adsorption required to produce a $5 \%$ change in diffraction efficiency for different values of initial phase difference, $\varphi$. Thus, when designing a transmission mode VG-based sensor, values of $\varphi=0,3.15,6.25,9.45$, etc radians must be avoided as extremely large changes in the grating phase due to gas adsorption will be required at these values in order to produce a measurable sensor response.

\section{Grating refractive index modulation, $\Delta n$}

The sensor response as a function of changes in $\Delta n$ and $d$ has been modelled for different values of initial VG $\Delta n$. The value of $\Delta n$ was varied
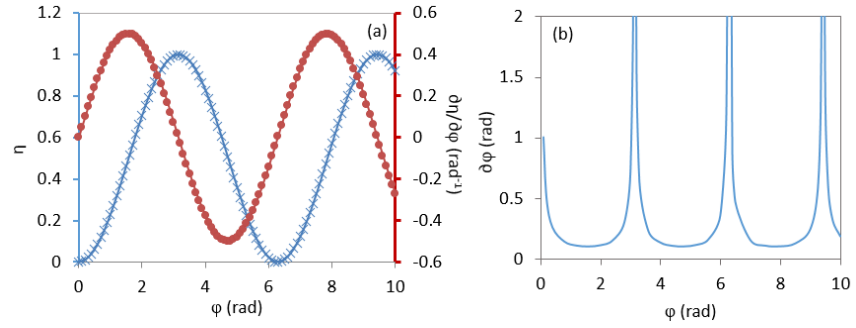

Fig. 5. For transmission mode VGs: (a) diffraction efficiency [left y-axis; $\times$ marker] and $\frac{\partial(\eta)}{\partial(\varphi)}$ [right y-axis; • marker] v.s. $\varphi(\mathrm{rad}) ;(\mathrm{b})$ the change in $\varphi$ (i.e. $\partial \varphi$ ) due to gas adsorption required to produce a $5 \%$ change in diffraction efficiency for different values of initial $\varphi$. 

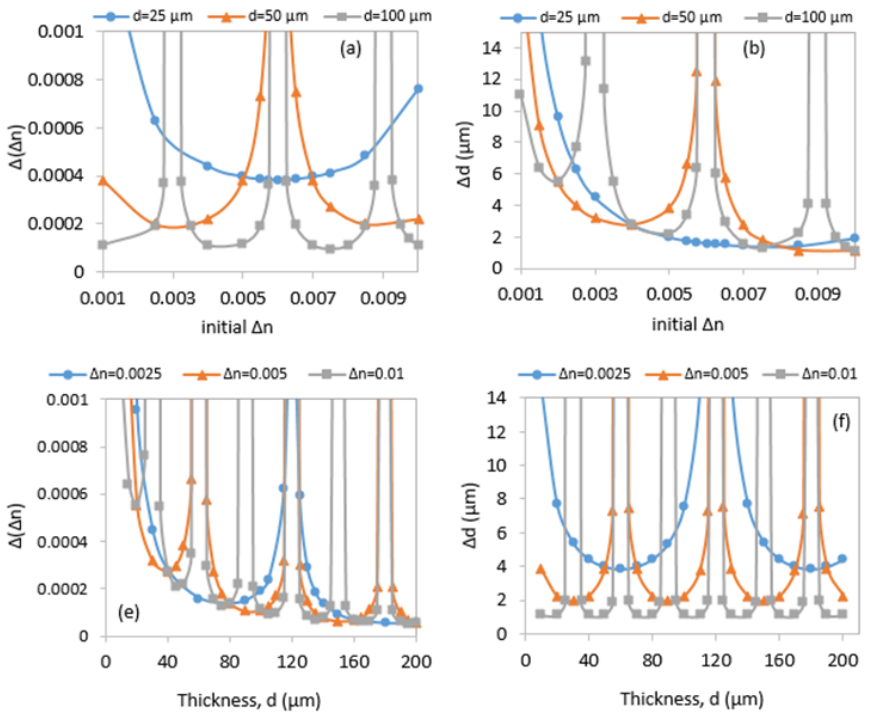

Fig. 6. $\Delta(\Delta n)$ and $\Delta d(\mu \mathrm{m})$ due to gas adsorption required to produce a $5 \%$ change in diffraction efficiency for different values of initial transmission VG (a,b) $\Delta n,(\mathrm{c}, \mathrm{d})$ spatial frequency, $(\mathrm{e}, \mathrm{f})$ thickness $(\mu \mathrm{m})$ and $(\mathrm{g}, \mathrm{h})$ reconstruction wavelength $(\mathrm{nm})$.

from 0.001 to 0.01 ; in reality this value will depend on the difference between the polymer matrix refractive index $\left(n_{p}\right)$ and the refractive index of the zeolite nanoparticles $\left(n_{z}\right)$ as well as the fraction of zeolite redistribution within the $V G$. This is a realistic $\Delta n$ range for transmission VGs recorded in a zeolite-doped acrylate photopolymer [21]. The modelling was carried out using eqns. 6 and 7 for spatial frequency = 1000 lines $/ \mathrm{mm}$ and $\lambda_{r}=633 \mathrm{~nm}$, for three different thicknesses: 25,50 and $100 \mu \mathrm{m}$.

Figs. 6(a) and (b) show the $\Delta(\Delta n)$ and $\Delta d$ due to gas adsorption required to produce a $5 \%$ change in grating diffraction efficiency for different initial values of grating $\Delta n$, respectively. A similar trend is observed for both modes of sensor operation; peaks tending to infinity are clearly observed for $d=50$ and $100 \mu \mathrm{m}$ in both cases. The positions of these peaks correspond to $\varphi=3.15,6.25,9.45$ etc. rad, as predicted by the general case in section 3A.1. For the lowest thickness studied, $d=$ $25 \mu \mathrm{m}$, no such peaks are observed for $\Delta n=0.001-0.01$, as an infinity peak $\varphi$ value has not yet been reached.

When designing a sensor, the value of $\Delta n$ will typically be fixed due to the constituent materials. The importance of careful selection of the other VG parameters to ensure optimum gas sensor operation (i.e. the lowest possible change in $\Delta n$ or $d$ due to gas adsorption required to produce a measurable change in sensor diffraction efficiency) is highlighted in Table 1, where the influence of initial VG thickness is used as an example. Table 1 shows optimum $d$ and $\Delta n$ combinations as well as the corresponding optimised sensor responses for the $\Delta(\Delta n)$ - and $\Delta d$ based sensors. E.g. for $100 \mu \mathrm{m}$ thick $\Delta(\Delta n)$-based sensors, four optimum $\Delta n$ values from $1 \times 10^{-3}$ to $1 \times 10^{-2}$ are identified which will produce a $5 \%$ change in diffraction efficiency resulting from a relatively small $\Delta n$ change due to gas adsorption of $1.11 \times 10^{-4}$. It is clear that a wide range of sensor materials with differing refractive index can be accommodated, which is advantageous for VG sensor design.

\section{Grating spatial frequency}

Next, the sensor response as a function of changes in $\Delta n$ and $d$ has been modelled for VG spatial frequency from $600-3000$ lines $/ \mathrm{mm}$. The calculations were carried out using values of $d=100 \mu \mathrm{m}$ and $\lambda_{r}=633$ $\mathrm{nm}$ for three different initial $\Delta n: 0.0025,0.005$ and 0.01 .
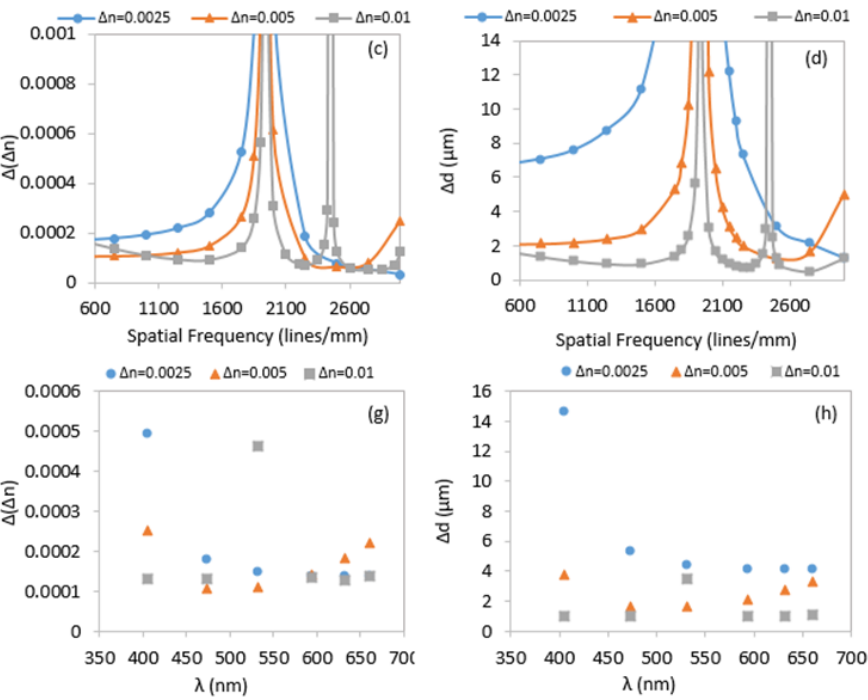

Figs. 6(c) and (d) show the $\Delta(\Delta n)$ and $\Delta d$ due to gas adsorption required to produce a $5 \%$ change in grating diffraction efficiency for different transmission VG spatial frequencies, respectively. As in section 3A.2, a similar trend is obtained for both sensor operational modes; infinity peaks are observed at 1950 lines $/ \mathrm{mm}$ in both cases for all three values of initial $\Delta n$, corresponding to $\varphi$ values of $3.15,6.25$ and 9.45 radians. For the largest $\Delta n$ value, 0.01 , a second peak is observed at 2450 lines $/ \mathrm{mm}$ corresponding to $\varphi=15.7$ radians.

The results show that spatial frequencies of $600-1750$ lines $/ \mathrm{mm}$ are generally suitable for fabrication of both $\Delta(\Delta n)$ - and $\Delta d$-based transmission VG sensors with initial $\Delta n=0.0025,0.005$ and 0.01 , as no infinity peaks (i.e. regions of poor device sensitivity) are present. Within this range, changes in sensor $\Delta n$ and $d$ due to gas adsorption required to produce a measurable $5 \%$ variation in sensor diffraction efficiency are in the order of $10^{-4}$ and $1-3 \mu \mathrm{m}$, respectively. The maximum achievable VG spatial frequency is limited mainly by the spatial resolution of the chosen sensor material.

\begin{tabular}{|c|c|c|c|}
\hline \multicolumn{4}{|c|}{ Table 1. Optimum $\Delta n$ values for the $\Delta(\Delta n)$ - and $\Delta d$-based sensors } \\
\hline \multirow{2}{*}{$\begin{array}{l}\text { Type of } \\
\text { sensor }\end{array}$} & \multirow{2}{*}{$\begin{array}{l}\text { Thickness } \\
(\mu \mathrm{m})\end{array}$} & \multirow[t]{2}{*}{ Optimum $\Delta n$} & Optimum \\
\hline & & & $\begin{array}{l}\text { Sensor response } \\
\text { i.e. } \Delta(\Delta n) / \Delta d \text { for } \Delta \eta \\
=5 \%\end{array}$ \\
\hline \multirow[b]{3}{*}{$\begin{array}{l}\Delta(\Delta n)- \\
\text { based }\end{array}$} & 25 & $6 \times 10^{-3}$ & $3.82 \times 10^{-4}$ \\
\hline & 50 & $\begin{array}{l}2.5 \times 10^{-3} \text { or } \\
8.5 \times 10^{-3}\end{array}$ & $1.98 \times 10^{-4}$ \\
\hline & 100 & $\begin{array}{c}1 \times 10^{-3} \text { or } \\
4 \times 10^{-3} \text { or } \\
7 \times 10^{-3} \text { or } \\
1 \times 10^{-2}\end{array}$ & $1.11 \times 10^{-4}$ \\
\hline \multirow{3}{*}{$\Delta d$-based } & 25 & $7.5 \times 10^{-3}$ & $1.38 \mu \mathrm{m}$ \\
\hline & 50 & $1 \times 10^{-2}$ & $1.10 \mu \mathrm{m}$ \\
\hline & 100 & $1 \times 10^{-2}$ & $1.11 \mu \mathrm{m}$ \\
\hline
\end{tabular}

\section{Grating thickness, $d$}

The sensor response as a function of changes in $\Delta n$ and $d$ has been modelled for different values of VG thickness, $d$, from 10-200 $\mu \mathrm{m}$, once again for three different values of initial VG $\Delta n$. Values for spatial frequency $=1000$ lines $/ \mathrm{mm}$ and $\lambda_{r}=633 \mathrm{~nm}$ were used for all calculations. The results for $\Delta(\Delta n)$ - and $\Delta d$-based sensors are shown in figs. 6(e) and (f). A large number of infinity peaks are present in the data, the number of which increase as $\Delta n$ is increased from 0.0025 to 0.005 to 0.01 . While it is difficult determine an "ideal" $d$ value for transmission VG-based sensors from these graphs, 50 and $75 \mu \mathrm{m}$ appear to offer "safe" $d$ options for 1000 lines/mm VG gratings as no infinity peaks are 
observed at these $d$ values. One advantage of transmission VG based sensors is that unlike for SRG-based sensors, it is relatively straightforward to fabricate VGs up to hundreds of microns thick, using a two beam holographic recording. Transmission VGs have an advantage over reflection VGs as the two recording beams are incident on the sample from the same side and their intensity is equally attenuated with sample depth, thus, larger thicknesses are possible.

\section{Reconstruction wavelength, $\lambda_{r}$}

The sensor response as a function of changes in $\Delta n$ and $d$ has been modelled for different commercially available $\lambda_{r}: 405,473,532,594$, 633 and $660 \mathrm{~nm}$. These calculations were carried out at $1000 \mathrm{lines} / \mathrm{mm}$, for $d=75 \mu \mathrm{m}$ and $\Delta n=0.0025,0.005$ and 0.01. Figs. 6(g) and (h) show the change in $\Delta n$ and $d$ due to gas absorption required to produce a change in $\eta$ of $5 \%$.

In both cases, different $\lambda_{r}$ are observed to be optimum for sensor response depending on the initial $\Delta n$ value. In some cases, the influence of $\lambda_{r}$ is extreme; e.g. for $\Delta n=0.0025 \mathrm{in} \mathrm{fig.} \mathrm{17,} \mathrm{the} \mathrm{required} \mathrm{change} \mathrm{in} \Delta n$ due to gas adsorption to produce a $5 \%$ change in diffraction efficiency is reduced by $70 \%$, from $4.9 \times 10^{-4}$ to $1.4 \times 10^{-4}$, as $\lambda_{r}$ is increased from $405 \mathrm{~nm}$ to $633 \mathrm{~nm}$. The variation observed is due to the oscillating Sine function in eqns. 6 and 7, which is governed by the relative size of the $\frac{\pi \Delta n d}{\lambda_{r} \cos \theta_{B}}$ term. This determines the phase difference between the zero and diffracted order, and therefore, the value of the VG diffraction efficiency as a function of $\Delta n$ and $d$. The significantinfluence of $\lambda_{r}$ on the response of both sensor types highlights that the relative size of the $\frac{\pi \Delta n d}{\lambda_{r} \cos \theta_{B}}$ term should be carefully considered when choosing $\lambda_{r}$.

\section{B. Modelling results for reflection mode VGs}

The sensor response (i.e. change in $\lambda_{r}$ as a function of change in $\Delta n$ and $d$ of the VG due to gas adsorption) of reflection mode VGs has been modelled using Kogelnik's Coupled Wave theory as described in section 2C.2. The influence of the initial $\Delta n$, spatial frequency, and thickness, $d$, on the sensor response has been investigated as a function of changes in both $\Delta n$ and $d$. The influence of reconstruction wavelength, $\lambda_{r}$, on sensor response was not modelled as reflection mode VGs are often reconstructed with white light, so that the wavelength shift due to analyte adsoption is clearly visible.

\section{Initial phase difference, $\varphi$}

In order to replicate the transmission mode VG study, we will first consider the general case for reflection mode VG-based gas sensors where the sensor output is varied due to changes in phase difference, $\varphi$. For the sake of comparison with the transmission mode
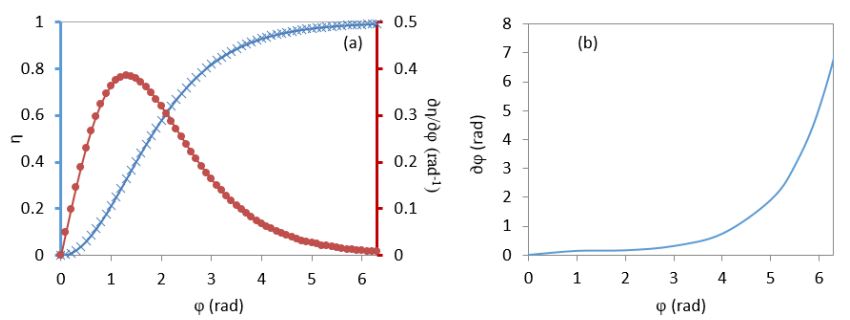

Fig. 7. For reflection mode VGs: (a) diffraction efficiency [left $y$-axis; $x$ ] and $\frac{\partial(\eta)}{\partial(\varphi)}$ [right y-axis; •] v.s. $\varphi(\mathrm{rad}) ;(\mathrm{b}) \partial \varphi$ due to gas adsorption required to produce a $5 \%$ change in diffraction efficiency for different values of initial $\varphi$.

VG devices, the sensor output is considered here to be a change in diffraction efficiency with gas exposure.
The diffraction efficiency of a reflection VG as a function of initial phase difference $\varphi$ given by eqn. 8 is shown in fig. 7(a). Unlike for transmission mode VGs there are no distinct peaks and troughs, however a plateau in diffraction efficiency is observed above $\varphi=5$ radians as diffraction efficiency approaches the maximum allowable value of 1 . In terms of sensor design, this is equivalent to one constant infinity peak. Fig. 7(a) shows that when the initial grating phase difference $\varphi$ value is exceeds 5 radians, the value of $\frac{\partial(\eta)}{\partial(\varphi)}$ approaches zero, and so the change in $\varphi$ required to produce a measurable change in diffraction efficiency tends to infinity. This is again clearly seen in fig. 7(b), which shows the change in $\varphi$ required to produce a 5\% change in diffraction efficiency for different values of initial $\varphi$. Thus, when designing a reflection mode VGbased sensor values of $\varphi>5$ radians should be avoided, as extremely large changes in the grating phase due to gas adsorption will be required in order to produce a measurable sensor response.

\section{Grating refractive index modulation, $\Delta \mathrm{n}$}

The dependence of the sensor response (i.e. change in $\lambda_{r}$ due to gas adsorption) of a $\Delta d$-based reflection VG on the initial value of grating $\Delta n$ from 0.001 to 0.01 (corresponding to zeolite nanoparticles with differing refractive index as well as fraction redistribution of zeolites within the grating) has been modelled using eqn. 11 . Values of spatial frequency $=4500$ lines $/ \mathrm{mm}$ and $\lambda_{r}=633 \mathrm{~nm}$ were used for these calculations. $\eta$ has been set at 30\%; the holographic sensor must be suitable for visual interrogation i.e. the reflection hologram must be bright and any colour change easily viewable. Previous studies of photopolymer-based reflection holograms have identified 30\% as a suitable value for $\eta$ [22]. From eqn. 8 it is clear that in order to maintain a minimum $\eta$ of $30 \%$, the initial value of $\Delta n$ will be varied, depending on the values for grating $d$ and $\theta$. E.g. for a spatial frequency of 4500 lines/mm and d values of $25-100 \mu \mathrm{m}$, the initial $\Delta n$ will vary between $2.0 \times 10^{-3}$ to $7.8 \times 10^{-3}$, in order to fulfil the $30 \%$ diffraction efficiency requirement.

Fig. 8(a) shows the change in $d$ due to gas adsorption required to produce shifts in $\lambda_{r}$ of $10 \mathrm{~nm}$ for initial $\Delta n$ from 0.001 to 0.01 . It is clearly seen that the sensor response improves as the initial VG $\Delta n$ value increases. Sub-micron changes in sensor $d$ due to gas adsorption are
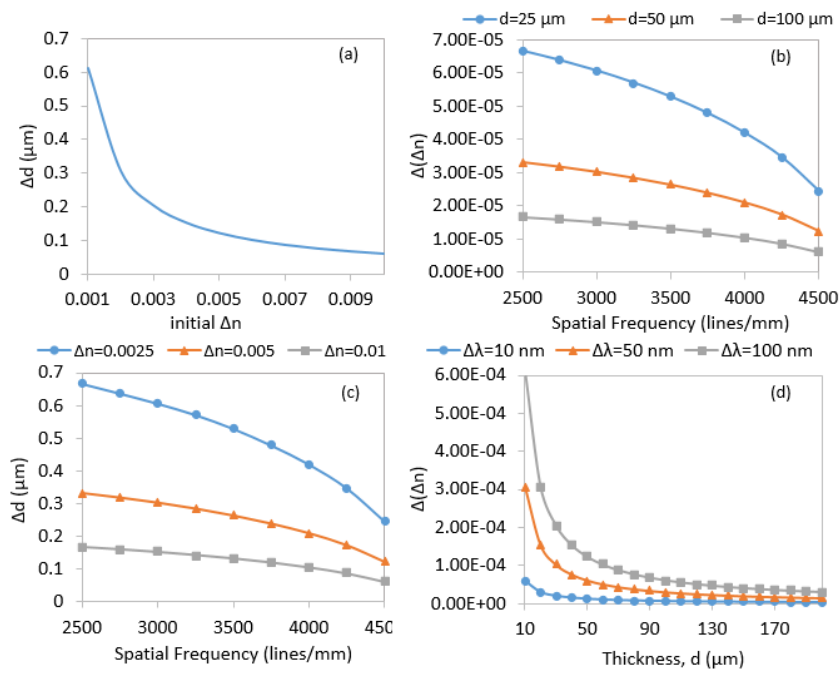

Fig. 8. $\Delta d(\mu \mathrm{m})$ and $\Delta(\Delta n)$ due to gas adsorption required to produce a $10 \mathrm{~nm}$ change in $\lambda_{r}$ for different values of initial reflection VG (a) $\Delta n$, $(b, c)$ spatial frequency and $(d)$ thickness $(\mu \mathrm{m})$.

capable of producing large, readily observable shifts in reconstruction wavelength, even for relatively low $\Delta n$ values of 0.001 . 


\section{Grating spatial frequency}

The sensor response as a function of changes in both $\Delta n$ and $d$ has been modelled for reflection VGs recorded at spatial frequencies in the range of $2500-4500$ lines $/ \mathrm{mm}$. This upper value was chosen as it represents the spatial frequency limit observed in the literature at which reflection holograms with sufficient brightness for visual sensors can be recorded in photopolymer materials [14, 22]. Photopolymers are of particular interest here as they are the only reported holographic material to be doped with zeolite nanoparticles for sensing applications [2,11-13]. $\eta=30 \%$ and $\lambda_{r}=633 \mathrm{~nm}$ were used for these calculations.

Fig. 8(b) shows that the $\Delta(\Delta n)$ due to gas adsorption required to produce a $10 \mathrm{~nm}$ shift in $\lambda_{r}$ for $\Delta(\Delta n)$-based sensors with initial $d$ of 25 , 50 and $100 \mu \mathrm{m}$ decreases by up to $65 \%$ with increasing spatial frequency. Similarly, fig. 8(c) shows that the $\Delta d$ due to gas adsorption required to produce an identical $10 \mathrm{~nm}$ shift in $\lambda_{r}$ for $\Delta d$-based sensors with initial $\Delta n$ of $0.0025,0.005$ and 0.01 decreases by up to $65 \%$ with increasing spatial frequency. This improved sensor response may be explained by the fact that as $\theta_{B}$ increases with increasing spatial frequency, the size of the $\cos \theta_{B}$ term in eqn 10 . decreases. The results also show that the sensor response is improved by the use of larger initial values of $d$ and $\Delta n$.

In contrast to the results for transmission mode VGs, there are no distinct infinity peaks present in the reflection mode VG sensor response as a function of spatial frequency. This is because for transmission mode VGs the diffraction efficiency is related to the $\varphi$ via a sine function as seen in eqn. 3 which passes through cycles of peaks and troughs. In contrast, for reflection mode VGs the diffraction efficiency is related to $\varphi$ via a hyperbolic tangent function as shown in eqn. 8 which does not undergo such oscillations.

\section{Grating thickness, $d$}

The sensor response of the $\Delta(\Delta n)$-based reflection VGs as a function of initial grating $d$ has been modelled using eqn. 10 for $d=10-200 \mu \mathrm{m}$. Values for spatial frequency $=4500$ lines $/ \mathrm{mm}, \lambda_{r}=633 \mathrm{~nm}$ and $\eta=30$ $\%$ were used for these calculations. It is clearly seen in fig. $8(\mathrm{~d})$ that the sensor response is improved as $d$ is increased. The change in $\Delta n$ due to gas exposure required to produce a $10 \mathrm{~nm}$ change in $\lambda$ decreases by an order of magnitude from $6 \times 10^{-5}$ to $6 \times 10^{-6}$ as $d$ is increased from 10 to $100 \mu \mathrm{m}$. The improved sensor response can be explained in practice by the fact that increased reflection VG thickness results in an increase in the number of layers of material with alternating refractive index (e.g. polymer and zeolite nanoparticles), thus providing increased number of analyte adsorption sites and improved sensor response to analyte adsorption.

\section{Discussion}

The sensor response of transmission and reflection mode VGs has been theoretically modelled and the optimum conditions for fabrication as well as the optimised sensor response for each configuration are outlined in Table 2.

Let us consider first the results for the $\Delta(\Delta n)$-based and $\Delta d$-based transmission mode VG sensors. For optimised values of spatial frequency, grating thickness and reconstruction wavelength, it is calculated that a $1 \%$ change in sensor readout diffraction efficiency will require a change in $\Delta n$ or $d$ due to gas adsorption in the order of $10^{-5}$ and 0.1-1.0 $\mu \mathrm{m}$ respectively. The exact value is dependent on the initial value of grating $\Delta n$, in particular for the $\Delta d$-based sensors, as seen in Table 2. The required change in $d$ decreases from $0.83 \mu \mathrm{m}$ to $0.10 \mu \mathrm{m}$ as the initial $\Delta n$ is increased from 0.0025 to 0.01 . For the $\Delta(\Delta n)$-based sensors, a similar change in $\Delta n$ due to gas adsorption of $\sim 2.1-2.8 \times 10^{-5}$ is required for all initial $\Delta n$. These initial values for $\Delta n$, spatial frequency and thickness identified to be optimum by the modelling for transmission mode VG sensors are readily achievable for holographic gratings recorded in zeolite-doped photopolymer media.

For the reflection mode VG sensors, it is calculated that a $10 \mathrm{~nm}$ change in sensor readout wavelength will require a change in $\Delta n$ or $d$ due to gas adsorption in the order of $10^{-6}$ and $0.1-0.2 \mu \mathrm{m}$ respectively. It is difficult to compare the sensor response of transmission and reflection mode VG devices due to the different readout mechanisms. However, if we assume a $1 \%$ change in readout diffraction efficiency is approximately equivalent to a $10 \mathrm{~nm}$ shift ( $1 \%$ from $660 \mathrm{~nm}$ ) in readout wavelength, the reflection mode devices are up to an order of magnitude more sensitive than the transmission VGs. This is due to the increased allowable VG spatial frequencies in the reflection regime, as in transmission mode the angle between the two recording beams is typically restricted by high Fresnel losses at large angles of incidence. This puts practical limitations on the maximum achievable spatial frequency, e.g. 3700 lines/mm and 5640 lines/mm in transmission and reflection mode, respectively, for a recording wavelength of 532 $\mathrm{nm}$.

An additional advantage of VG sensors is that they can be fabricated in reflection mode, allowing for visual readout via a colour change in the presence of a gas. However, as seen in Table 2, only relatively small changes in both $\Delta n$ and $d$ are required to produce shifts in the sensor

\begin{tabular}{|c|c|c|c|c|c|c|}
\hline & & $\Delta n$ & $\begin{array}{l}\text { Spatial Frequency } \\
\text { (lines } / \mathrm{mm} \text { ) }\end{array}$ & $d(\mu m)$ & $\lambda_{r}(\mathrm{~nm})$ & $\begin{array}{c}\text { Sensor response } \\
\text { i.e. } \Delta(\Delta n) \text { or } \Delta d \text { for } \Delta \eta=1 \%\end{array}$ \\
\hline \multirow{7}{*}{ Transmission } & $\Delta(\Delta n)$-based & 0.0025 & \multirow{3}{*}{1000} & \multirow{3}{*}{75} & 594 & $2.75 \times 10^{-5}$ \\
\hline & & 0.005 & & & 473 & $2.13 \times 10^{-5}$ \\
\hline & & 0.01 & & & 633 & $2.55 \times 10^{-5}$ \\
\hline & \multirow[t]{4}{*}{$\Delta d$-based } & 0.0025 & \multirow{3}{*}{1000} & \multirow{3}{*}{75} & 594 & 0.83 \\
\hline & & 0.005 & & & 473 & 0.32 \\
\hline & & 0.01 & & & 633 & 0.19 \\
\hline & & $\Delta n$ & $\begin{array}{l}\text { Spatial Frequency } \\
\text { (lines } / \mathrm{mm} \text { ) }\end{array}$ & & & $\begin{array}{c}\text { Sensor response } \\
\text { i.e. } \Delta(\Delta n) \text { or } \Delta d \text { for } \Delta \lambda=10 \mathrm{~nm}\end{array}$ \\
\hline \multirow{6}{*}{ Reflection } & \multirow[t]{3}{*}{$\Delta(\Delta n)$-based } & & \multirow{3}{*}{4500} & \multicolumn{2}{|c|}{25} & $2.45 \times 10^{-5}$ \\
\hline & & - & & \multicolumn{2}{|c|}{50} & $1.25 \times 10^{-5}$ \\
\hline & & & & \multicolumn{2}{|c|}{100} & $6.14 \times 10^{-6}$ \\
\hline & \multirow[t]{3}{*}{$\Delta d$-based } & 0.0025 & \multirow{3}{*}{4500} & \multirow{3}{*}{\multicolumn{2}{|c|}{-}} & 0.246 \\
\hline & & 0.005 & & & & 0.123 \\
\hline & & 0.01 & & & & 0.061 \\
\hline
\end{tabular}


readout for both transmission and reflection mode VG devices. This may present issues when developing a sensor that responds to a change in a single parameter only, as highlighted in section 2B. This is an ongoing challenge for sensor design in general, and will likely be addressed by further development of sensor materials with enhanced structural and chemical properties.

By comparison with Part 1 [1], the transmission and reflection mode VG-based sensors are shown to be up to three orders of magnitude more sensitive than the thin and thick SRG-based sensors: the optimum sensitivity achieved in $\Delta n$-based thin and thick SRG-based sensors is $4.9 \times 10^{-3}$ and $2.7 \times 10^{-4}$, respectively, in comparison to $2.8 \times$ $10^{-5}$ and $6.1 \times 10^{-6}$ for $\Delta n$-based transmission and reflection mode VG-based sensors, respectively. The difference in sensitivity is largely due to the different allowable physical parameters; larger $\Delta \mathrm{n}$ values are achievable with SRG-based devices, whereas increased spatial frequencies and thicknesses are possible for the VG-based sensors. The optimum fabrication conditions for each configuration are given in Table 3. As in Part 1 for the SRG-based sensors, a main conclusion from the study is that the reconstruction wavelength used for sensor readout has a significant impact on the sensitivity of the transmission mode sensor system and should be given careful consideration when designing a sensor system which uses a single wavelength probe beam.

\begin{tabular}{|c|c|c|c|c|c|}
\hline \multicolumn{6}{|c|}{$\begin{array}{l}\begin{array}{l}\text { Table 3. Optimum* fabrication conditions for SRG and VG } \Delta \text {-based } \\
\text { sensors }\end{array} \\
\end{array}$} \\
\hline & $\Delta n$ & $\begin{array}{l}S F \\
\text { (lines/ } \\
\mathrm{mm} \text { ) }\end{array}$ & $\begin{array}{l}d \\
(\mu m)\end{array}$ & $\begin{array}{l}\lambda_{r} \\
(n m)\end{array}$ & $\begin{array}{l}\Delta(\Delta n) \text { for } \Delta \eta= \\
1 \% \\
\left({ }^{* *} \Delta \lambda=10 n m\right)\end{array}$ \\
\hline Thin SRG & 0.15 & 500 & 1 & 405 & $4.9 \times 10^{-3}$ \\
\hline Thick SRG & 0.15 & 1500 & 4.5 & 405 & $2.7 \times 10^{-4}$ \\
\hline Transmission VG & 0.0025 & 1000 & 75 & 594 & $2.8 \times 10^{-5}$ \\
\hline $\begin{array}{l}\text { Reflection } \mathrm{VG}^{* *} \text {, } \\
30 \% \mathrm{DE}\end{array}$ & 0.0004 & 4500 & 100 & 633 & $6.1 \times 10^{-6}$ \\
\hline
\end{tabular}

* within the studied set of parameters

\section{CONCLUSIONS}

Theoretical modelling and design of VG structures recorded in zeolite composite materials for gas sensing applications has been carried out. The sensor response of transmission and reflection mode VGs was modelled using Kogelnik's coupled wave theory. This study was carried out for sensors operating based on changes in both $\Delta n$ and $d$ due to gas adsorption. The influence of a range of parameters on the sensor response of $\Delta(\Delta n)$ - and $\Delta d$-based VG sensors has been studied, namely grating phase, zeolite nanoparticle refractive index, grating spatial frequency, grating thickness and reconstruction wavelength. From this, the optimum fabrication conditions and coresponding sensitivities for a range of VG-based gas sensors have been identified. For optimised transmission mode VG-based sensors, changes in $\Delta n$ and $d$ due to analyte adsorption in the order of $10^{-5}$ and $0.2-1 \mu \mathrm{m}$ respectively are required to produce a $1 \%$ change in sensor readout diffraction efficiency. In comparsion, changes in $\Delta n$ and $d$ due to analyte adsorption in the order of $10^{-6}$ and $0.1-0.2 \mu \mathrm{m}$ respectively are required to produce a $10 \mathrm{~nm}$ change in readout wavelength for optimised reflection mode VG-based sensors. The importance of carrying out theoretical modelling in the design of VG-based sensors prior to fabrication has been highlighted.

Funding information. Dublin Institute of Technology's Arnold F Graves Postdoctoral Fellowship Programme.
1. D. Cody and I. Naydenova, "Theoretical modeling and design of photonic structures in zeolite nanocomposites for gas sensing. Part I: surface relief gratings," J. Opt. Soc. Am. A 34, 2110-2119 (2017).

2. M. Zawadzka, T. Mikulchyk, D. Cody, S. Martin, E. Mihaylova, A. K. Yetisen, J. L. Martinez-Hurtado, H. Butt, H. Awala, S. Mintova, S. H. Yun, and I. Naydenova, "Photonic Materials for Holographic Sensing" in "Photonic Materials for Sensing, Biosensing, and Display Devices", Springer (2015).

3. A. G. Mayes, J. Blyth, M. Kyröläinen-Reay, R. B. Millington, and C. R. Lowe, "A holographic alcohol sensor", Anal. Chem. 71(16), 3390-3396 (1999).

4. S. Kabilan, J. Blyth, M. C. Lee, A. J. Marshall, A. Hussain, X.-P. Yang and C. R. Lowe, "Glucose-sensitive holographic sensors", J. Mol. Recogn. 17(3), 162-166 (2004).

5. B. M. González, G. Christie, C. A.B. Davidson, J. Blyth, and C.R. Lowe, "Divalent metal ion-sensitive holographic sensors", Anal. Chim. Acta 528(2), 219-228 (2005).

6. A. J. Marshall, J. Blyth, C. A. B. Davidson, and C. R. Lowe, "pH-sensitive holographic sensors", Anal. Chem. 75(17), 4423-4431 (2003).

7. T. Mikulchyk, S. Martin, and I. Naydenova, "Humidity and temperature effect on properties of transmission gratings recorded in PVA/AA-based photopolymer layers", J. Opt. 15(10) (2013).

8. C. R. Lowe, J. Blyth, and A. P. James, "Interrogation of a sensor", US 20080094635 A1 (2008), 24th April 2008.

9. E. Mihaylova, D. Cody, I. Naydenova, S. Martin, and V. Toal, "A Holographic Recording Composition", WO2016001108, 7th January 2016.

10. E. Leite, I. Naydenova, S. Mintova, L. Leclercq, and V. Toal, "Photopolymerisable Nanocomposites for Holographic Recording and Sensor Application", Appl.Opt. 49, 3652-3660 (2010).

11. Dongyao Mao, Yaohui Geng, Hongpeng Liu, Ke Zhou, Lihong Xian, and Dan Yu, "Two-way shift of wavelength in holographic sensing of organic vapor in nanozeolites dispersed acrylamide photopolymer", Appl. Opt. 55(23), 6212-6221 (2016).

12. Dan Yu, Hongpeng Liu, Dongyao Mao, Yaohui Geng, Weibo Wang, Liping Sun, and Jiang Lv, "Enhancement of spectrum strength in holographic sensing in nanozeolites dispersed acrylamide photopolymer", Opt. Express 23(22), 29113-29126 (2015).

13. V. K. S. Hsiao, W. D. Kirkey, F. Chen, A. N. Cartwright, P. N. Prasad, and T.J. Bunning, "Organic Solvent Vapor Detection Using Holographic Photopolymer Reflection Gratings" Adv. Mater. 17(18), 2211-2214 (2005).

14. I. Naydenova, J. Raghavendra, V. Toal, S. Martin, "A Visual Indication of Environmental Humidity using a Color Changing Hologram Recorded in a Self-developing Photopolymer" Appl. Phys. Lett. 92, 031009 (2008).

15. J. L. Martínez-Hurtado, C. A. Davidson, J. Blyth, and C. R. Lowe, "Holographic detection of hydrocarbon gases and other volatile organic compounds" Langmuir 26(19), 15694-15699 (2010).

16. M. Moothanchery, I. Naydenova, and V. Toal, "Studies of shrinkage as a result of holographic recording in acrylamide-based photopolymer film", Appl. Phys. A 104(3), 899-902 (2011).

17. W. R. Klein and B. D. Cook, "Unified approach to ultrasonic light diffraction", IEEE Trans. Sonics Ultrason. 14(3), 123 - 134 (1967).

18. M.G. Moharam and L. Young, "Criterion for Bragg and Raman-Nath diffraction regimes", Appl. Opt. 17 (11), 1757-1759 (1978).

19. W. L. Bragg, "The Diffraction of Short Electromagnetic Waves by a Crystal". Proc. Camb. Philos. Soc. 17: 43-57 (1913).

20. H. Kogelnik, "Coupled wave theory for thick hologram gratings", The Bell Syst. Tech. J. 48(9), 2909-2947 (1969).

21. D. Cody, E. Mihaylova, L. O'Neill, T. Babeva, H. Awala, R. Retoux, S. Mintova. and I. Naydenova, "Effect of zeolite nanoparticles on the optical properties of diacetone acrylamide-based photopolymer" Opt. Mater. 37, 181-187 (2014).

22. D. Cody, S. Gribbin, E. Mihaylova, and I. Naydenova, "Low-toxicity photopolymer for reflection holography", ACS Appl. Mater. Interfaces 8 (28), 18481-18487 (2016).

\section{References:}

